\title{
Time Resolved Electron Energy Loss Spectroscopy as a Tool for Controlling and Monitoring the Early Stages of Electron Beam Induced Transformations.
}

\author{
S. Trasobares ${ }^{* *}$, O. Stéphan ${ }^{*}$, C. Colliex ${ }^{*}$ \\ ** Presently at Department of Material Science \& Engineering at Rensselaer Polytechnic Institute, \\ Troy, NY 12180-3590, USA. \\ *Laboratoire de Physique des Solides, Building 510, Université Paris Sud, 91405 Orsay, France.
}

Spatially Resolved Electron Energy Loss Spectroscopy (EELS) in the context of a Scanning Transmission Electron Microscope (STEM) has been demonstrated to be a powerful technique for studying the chemistry and electronic structure of materials at the nanometer scale. Most recent developments on the acquisition system have highly improved the sensitivity of the technique, as clearly demonstrated by selective atoms imaging [1]. The high detention efficiency of the charged coupled device (CCD) camera detector offers new possibilities to the EEL spectroscopy. With the new system, the required acquisition time for recording a satisfactory signal has been brought down to 10 to a few $100 \mathrm{~ms}$ for the core loss region (i.e. on the $\mathrm{C}$ or $\mathrm{N}$ Kedge). These improvements provide the possibility of studying the early stages of beam induced specimen transformation, by monitoring the Energy Loss Near Edge Structures (ELNES) changes using Time Resolved EELS (chrono-spectroscopy mode). Moreover the combination of Time and Spatially Resolved EELS with adapted mathematical methods, like Multivariate Statistical Analysis (MSA), allows us to control and monitor in situ in the electron microscope the electron beam induced transformations. In the chrono-spectroscopy mode, a probe of $0.5 \mathrm{~nm}$ in diameter with a current of $c a 0.15-0.20 \mathrm{nA}$ is fixed at a specific location on the specimen and a series of spectra are recorded with the dose as variable (with roughly dose $=$ time $\times 10^{9} \mathrm{e}^{-} / \mathrm{nm}^{2} \mathrm{~s}$ ). In this paper, we illustrate this technique by studying the beam-induced processes in two different systems:

Electron beam induced processes on melamine.

Melamine is highly sensitive to radiation damage, visual changes are observed in the analysed area as soon as the electron beam is focused on the system. Time Resolved EELS data reveals $\mathrm{C}$ and $\mathrm{N}$ losses during the process, with a $40 \%$ decrease on the N/C ratio being observed. Monitoring ELNES shows that a strong $\mathrm{p}^{*}$ peak at the carbon edge, which is observed at the beginning of the processs, strongly decreases when the total applied dose is approximately $10^{11} \mathrm{e}^{-} / \mathrm{nm}^{2}$. For the $\mathrm{N} \mathrm{K}$ edge, the situation is more complex, the appearance and vanishing of several peaks at the onset of the $\mathrm{N} \mathrm{K}$ edge are observed during the process. A clear peak at $401.1 \mathrm{eV}$, which has being associated to the presence of $\mathrm{N}_{2}$ gas, is observed when the applied dose is $4 \times 10^{8} \mathrm{e}^{-} / \mathrm{nm}^{2}$. From the comparison of experimental data with simulated spectra obtained using molecular orbital theory, we suggest that the amino groups are first eliminated and $\mathrm{N}_{2}$ gas is released as one of the decomposition products. Then the degradation of the molecule occurs through a reduction of the carbon-nitrogen double bonds either by addition of hydrogen or by bond cleavage. If the irradiation is maintained over longer periods the formation of different $\mathrm{CN}$ fragments and polymerisation products may happen [2].

Controlling and monitoring electron beam induced process on nitrogen doped nanotubes.

Spatially resolved EELS on carbon nanotubes produced by catalytic pyrolysis of camphor provides the first experimental evidence of trapping $\mathrm{N}_{2}$ in the cavity of the nanotube during the synthesis process. Performing chrono-spectroscopy on these nano-structures, we have monitored and controlled the produced changes under electron beam irradiation. $\mathrm{CN}_{\mathrm{x}}$ nano-islands $(20 \mathrm{~nm}$ in 
diameter) have been regularly produced all along the nanotube by puncturing the walls of the nanotube, releasing most of the trapped gas and gradually incorporating the remained nitrogen into the previously amorphised carbon system, see Fig1 [3].

[1] K. Suenaga et al., Science 290 (2000) 2280.

[2] S. Trasobares et al., Chem Phys J, submitted (2002).

[3] S. Trasobares et al., Eur. Phys. J. B 22 (2001) 117.

[4] M. Tencé is thanked for the microscope and software developments. K.W. Hsu, D.R.M. Walton and H.W. Kroto at Sussex Univ. are acknowledged for the nanotubes samples preparation and for fruitful discussion. L. Petterson, C. Kolczewski, N. Borglund and S. Csillag at Stockholm Univ. are thanked for the theoretical calculations on the melamine decomposition products. Financial support has been provided by an EU-TMR network.

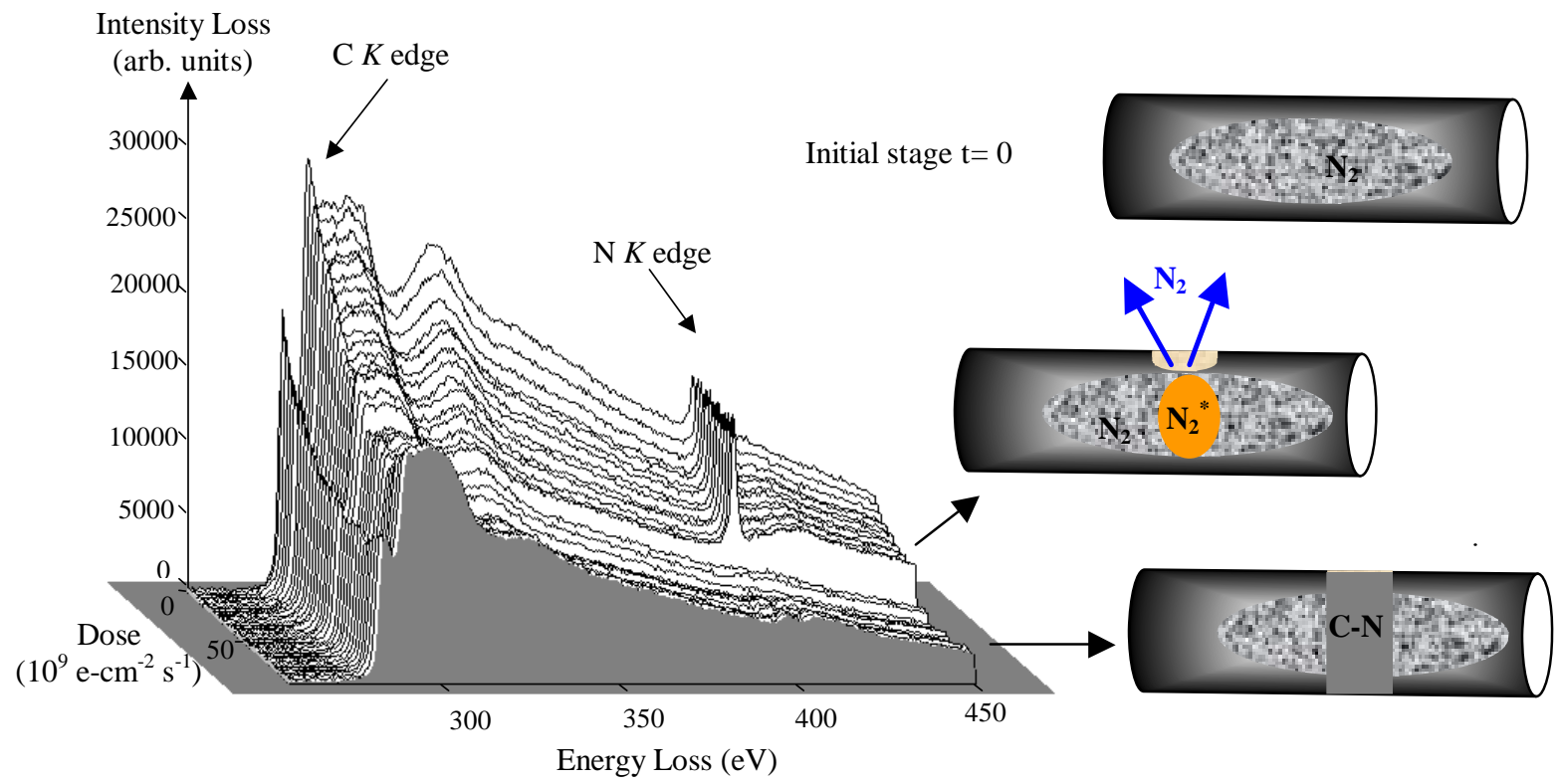

FIG.1. Time sequence EELS spectra on nanotubes containing gas and the electron beam induced process scheme. 